J. Dairy Sci. 95:4519-4525

http://dx.doi.org/10.3168/jds.2011-5119

(C) American Dairy Science Association ${ }^{\circledR}, 2012$.

\title{
Development of colonic microflora as assessed by pyrosequencing in dairy calves fed waste milk
}

\author{
T. S. Edrington, ${ }^{\star 1}$ S. E. Dowd,† R. F. Farrow, ${ }^{\star}$ G. R. Hagevoort, $¥$ T. R. Callaway, ${ }^{\star}$ R. C. Anderson, ${ }^{*}$ \\ and D. J. Nisbet* \\ ${ }^{*}$ Food and Feed Safety Research Unit, Southern Plains Agricultural Research Center, USDA-ARS, College Station, TX 77845, and \\ †Research and Testing Laboratory, Lubbock, TX 79410 \\ $\ddagger$ Agricultural Experiment Station, New Mexico State University, Clovis, NM 88101
}

\section{ABSTRACT}

The objective of the current study was to examine the effect of pasteurization of waste milk, used to feed dairy calves, on the bacterial diversity of their lower gut. Using $16 \mathrm{~S}$ rDNA bacterial tag-encoded FLX amplicon pyrosequencing, fecal samples from dairy calves, ages 1 wk to 6 mo old and fed either pasteurized or nonpasteurized waste milk, were analyzed for bacterial diversity. Calves were maintained on 2 separate farms and, aside from how the waste milk was treated, were housed and cared for similarly. Fifteen calves were sampled from each age group $(1,2$, and $4 \mathrm{wk}$, and 2,4 , and 6 mo of age; $\mathrm{n}=90$ samples per milk treatment, 180 total samples) on each farm via rectal palpation and the samples shipped and frozen before analysis. In general, bacterial diversity, as represented by the total number of different species, was greater for the calves fed pasteurized waste milk at all ages (except 1 wk of age) and increased with increasing age in both treatments. Proteobacteria, Bacteroidetes, and Firmicutes were the predominant phyla. Differences in phyla and class were observed among treatments and age of calf but with no consistent trends. Salmonella were detected in 9 out of $14(64 \%)$ of the 1-wk-old calves fed nonpasteurized milk. Treponema, an important beneficial bacterium in cattle rumen, was more prevalent in the pasteurized waste milk-fed animals and became higher in the older animals from this group. Escherichia-Shigella were detected among treatments at all ages, and highest at 1 wk of age, averaging approximately 21 and $20 \%$ of all bacteria for calves fed pasteurized and nonpasteurized waste milk, respectively, and decreasing as calves aged (2.6 and 1.3\%). The consistent detection of Salmonella in the younger animals fed nonpasteurized milk and its absence in all other groups is an important finding related to this feeding practice.

Received November 3, 2011.

Accepted April 4, 2012.

${ }^{1}$ Corresponding author: tom.edrington@ars.usda.gov
Key words: bacterial diversity, Salmonella, waste milk, pasteurization

\section{INTRODUCTION}

Although the specifics of calf management programs on commercial dairy operations in the United States vary, not only among regions but also among farms within a geographical location, the general procedures are the same. Calves are removed from their dams soon after birth, administered colostrum, and housed in individual hutches. Feeding programs typically involve twice-daily feeding of whole milk, milk replacer, or a combination of the two, along with water and a starter feed provided. Calves are generally weaned from the milk diet anywhere from 4 to 8 wk of age, once intake of the starter feed is deemed sufficient, and are often moved from individual to group pens at this same time.

The size of modern commercial dairies had increased substantially in the past 20 yr (USDA-NASS, 2002), particularly in the western United States, with farms of 2,000 to 3,000 head relatively common. This increase in herd size has also resulted in an increase in nonsaleable (discard or waste) milk produced. Waste milk is produced from cows in the first few milkings of the lactation cycle or from cows treated with antibiotics, often for mastitis, and cannot be sold for human consumption (Chardavoyne, et al., 1979). The amount of waste milk produced is substantial and ranges from 22 to 62 $\mathrm{kg}$ per cow per year (Blosser, 1979). To reduce some of this economic loss, dairy producers use this milk to feed calves. The 2002 National Animal Health Monitoring System (NAHMS) Dairy Survey reported that $87.2 \%$ of dairy farms in the United States use waste milk to feed their calves (USDA-NASS, 2002). Research has demonstrated that growth rates and the incidence of scours were similar among calves fed waste milk as compared with milk-fed controls (Chardavoyne et al., 1979; Keys et al., 1980; Kesler, 1981); however, waste milk can expose young calves to various pathogens. In an effort to reduce this risk of transmission, producers are pasteurizing waste milk before calf feeding. Benefits 
of on-farm pasteurization include reduced diarrhea and pneumonia and improved weight gains (Jamaluddin et al., 1996). Pasteurization also kills bacteria such as $M y$ cobacterium paratuberculosis (responsible for Johne's disease), Salmonella, and Mycoplasma (Stabel et al., 2004), if present in the milk.

Salmonella is considered an important pathogen in dairy calves and a significant causative factor of neonatal calf scours. Previous research in our laboratory indicated that fecal shedding of Salmonella, and in particular multidrug-resistant Salmonella, was most prevalent in young calves (preweaning) and cows in the hospital pen (Edrington et al., 2008). This suggests that Salmonella is opportunistic and tends to thrive in an immature or disturbed gastrointestinal microflora, such as would be found in these 2 subpopulations of dairy cattle. Pasteurization of waste milk, if done properly, will kill Salmonella (Stabel et al., 2004) and significantly decreases the number of total bacteria (Ruzante et al., 2008). On the other hand, pasteurization would also kill beneficial bacteria that may be contained in the waste milk, species that might enhance gastrointestinal maturity of the gut and aid in the prevention of Salmonella colonization. Therefore, the objective of the current experiment was to examine bacterial diversity and Salmonella populations in dairy calves from $1 \mathrm{wk}$ to 6 mo of age and fed either pasteurized or nonpasteurized waste milk.

\section{MATERIALS AND METHODS}

\section{Animals and Sample Collection}

Fecal samples analyzed in this research were collected on 2 large commercial dairy farms $(>3,000$ head) in the southwestern United States in April 2008. Calves were sampled on each farm representing 6 age groups: $1 \mathrm{wk}$, 2 wk, and 1, 2, 4, and 6 mo of age. Both farms used waste milk to feed the calves before weaning, one farm pasteurizing the milk before feeding and the other using nonpasteurized waste milk. Other than pasteurization of the waste milk, management, care and housing of the calves were similar on both farms. Fecal samples (approximately $20 \mathrm{~g}$ ) were collected via rectal palpation or from freshly voided fecal pats from 15 animals per age group on each farm ( $\mathrm{n}=90$ samples/farm; 180 total samples). Fecal samples were collected into sterile palpation sleeves, placed on ice, and shipped to our laboratory in College Station, Texas. A portion of the fecal sample $(\sim 2 \mathrm{~g})$ was placed in a $15-\mathrm{mL}$ conical tube and frozen before shipping to the Research and Testing Laboratory (Lubbock, TX) for the bacterial tagencoded FLX amplicon pyrosequencing (bTEFAP) processing as described below.

\section{Bacterial Tag-Encoded FLX Amplicon Pyrosequencing Analysis}

Fecal samples were subjected to bacterial tag-encoded FLX amplicon pyrosequencing, as described previously (Dowd et al., 2008; Smith et al., 2010; Ishak et al., 2011). Samples were processed based upon FLX (nontitanium chemistry), generating an average read length of $248 \mathrm{bp}$. Extraction of total genomic DNA from the fecal samples was accomplished using a QIAamp stool DNA mini kit per the manufacturer's recommendations (Qiagen Inc., Valencia, CA). The DNA samples were quantified using a Nanodrop spectrophotometer (Nyxor Biotech, Paris, France). Custom software written in C\# within Microsoft.NET (Microsoft Corp., Seattle, WA) development environment was used for all postsequencing processing (Dowd et al., 2008; Smith et al., 2010; Ishak et al., 2011). Sequences were Q20 trimmed, tags and primers removed, chimeras removed, short reads $<220$ removed, plastid and mitochondrial sequences removed, sequences with degenerate bases removed, and sequences with homopolymers $>5 \mathrm{bp}$ removed. Sequence data was then analyzed as has been described previously to derive taxonomic data (Dowd et al., 2008; Smith et al., 2010; Ishak et al., 2011) and diversity estimates calculated using mothur (Schloss et al., 2009) and UniFrac analysis (Lozupone et al., 2011) were performed as described previously (Pitta et al., 2010). Classification was performed using the BLASTn program (available at http://www.ncbi.nlm. nih.gov/blast/) as described previously (Smith et al., 2010; Ishak et al., 2011).

\section{Statistics}

Statistics were performed on the bacterial diversity data using NCSS 2007 software (NCSS LLC, Kaysville, UT). Fecal Salmonella prevalence was subjected to chisquared analysis using the PROC FREQ procedure of SAS Institute Inc. (Cary, NC). Differences were considered significant at $P<0.05)$ unless otherwise stated. The data for individual animals was compiled into age groups and farms by pooling the sequence data from each individual animal and processing the data together. Following this, dual dendrograms were constructed in NCSS 2007 using Ward's minimum variance clustering and Manhattan distances (NCSS LLC). Principle component analysis was based upon UniFrac methods.

\section{RESULTS}

In general, bacterial diversity, as represented by the total number of different characterized and noncharacterized species, increased with increasing calf age and 


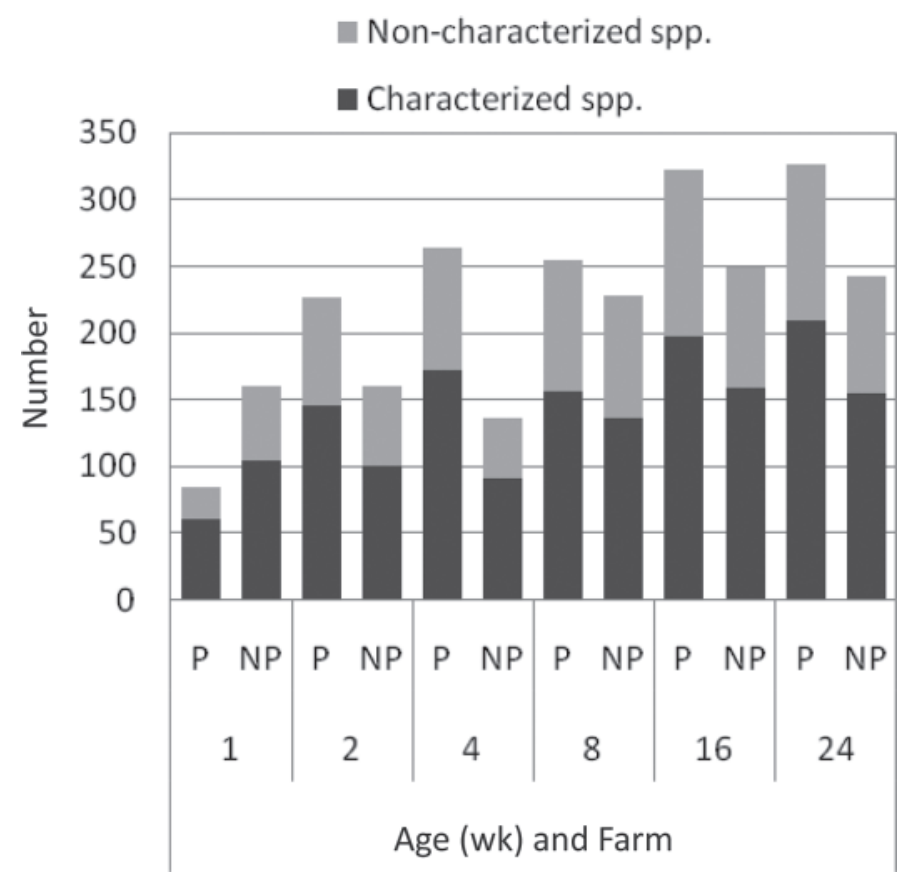

Figure 1. Bacterial diversity, as measured by total number of characterized and noncharacterized species, in dairy calves 1 to 24 wk of age and fed either pasteurized $(\mathrm{P})$ or nonpasteurized (NP) waste milk.

was greater for the calves fed pasteurized waste milk at all ages, with the sole exception of 1-wk-old calves (Figure 1). Chao1 values (estimate of the true species diversity of a sample) and rarefaction estimates were calculated at $3 \%$ divergence to estimate the number of species for each group of animals. The diversity increased with age. The pasteurized milk-fed animals started with the lowest diversity compared with the nonpasteurized milk-fed animals (Chao1 162 vs. 215); however, by wk 2 , the diversity estimators were consistently higher in the pasteurized milk-fed groups (Table 1). A more specific comparison of the predominant phyla is presented in Table 2. Proteobacteria accounted for approximately 20 to $30 \%$ of the total phyla at 1 and 2 wk of age, decreasing by 4 wk of age and remaining at a low percentage through the remaining collections. The number of Spirochetes in the nonpasteurized milk-fed groups steadily increased with age, becoming significantly higher at 6 mo of age $(P<0.001)$ when compared with all groups from the pasteurized milkfeeding farm. Bacteroidetes and Firmicutes were the most prevalent phyla, regardless of type of milk fed and were the predominant 2 phyla by 4 wk of age and older. Actinobacteria were relatively low in number (less that $10 \%$ of total phyla) at all ages on both farms. At $4 \mathrm{wk}$, Bacteroidetes were significantly greater in number in the group fed pasteurized milk.

Figure 2 presents a broad overview of the 50 genera that were the most abundant among the combined group analysis (average percentage across all groups or animals). The level of relatedness was closest at 1 and 2 wk of age (both farms; more similarity in the calves fed nonpasteurized milk), at $4 \mathrm{wk}$ and 2 mo of age (both farms), and at 4 and 6 mo of age (each farm). This indicates that the farms have distinct microbial populations as animals mature, possibly related to the early dietary differences. Highlighted in the dendrogram on the right $\mathrm{y}$-axis are those genera that contributed strongly to the clustering. Prevotella was a strong population in the 4- and 6-mo-old group from farm B and Akkermansia population was notably larger in 4- and 6-mo-old calves from farm A (also seen in the 2-mo-old group). The Shigella-Escherichia population was the notable population in both farms for the younger animals and an absence of Collinsella abundance distinguished the 2-mo-old animals in both groups.

Based upon UniFrac weighted principal component analysis, we evaluated 1-wk, 4-wk, 2-mo, and 6-mo groups (Figure 3 ). Calves at $1 \mathrm{wk}$ of age had similar microbial populations that were not significantly differ-

Table 1. Diversity analysis using mothur software (Schloss et al., 2009), providing Chao1 (estimate of the true species diversity of a sample) and rarefaction estimations at $3 \%$ divergence in fecal samples collected from dairy calves, 1 wk to 6 mo in age, and fed either pasteurized (A) or nonpasteurized (B) waste milk

\begin{tabular}{lccc}
\hline Group & Sequences (no.) & Chao1 $3 \%$ (no.) & Rarefaction 3\% (no.) \\
\hline A 1 wk & 19,585 & 162 & 92 \\
A 2 wk & 26,725 & 335 & 163 \\
A 4 wk & 17,289 & 584 & 289 \\
A 2 mo & 11,483 & 590 & 295 \\
A 4 mo & 17,998 & 689 & 340 \\
A 6 mo & 46,936 & 823 & 363 \\
B 1 wk & 18,560 & 215 & 112 \\
B 2 wk & 19,554 & 310 & 142 \\
B 4 wk & 4,007 & 496 & 194 \\
B 2 mo & 13,034 & 596 & 224 \\
B 4 mo & 15,753 & 579 & 271 \\
B 6 mo & 17,696 & & 257 \\
\hline
\end{tabular}




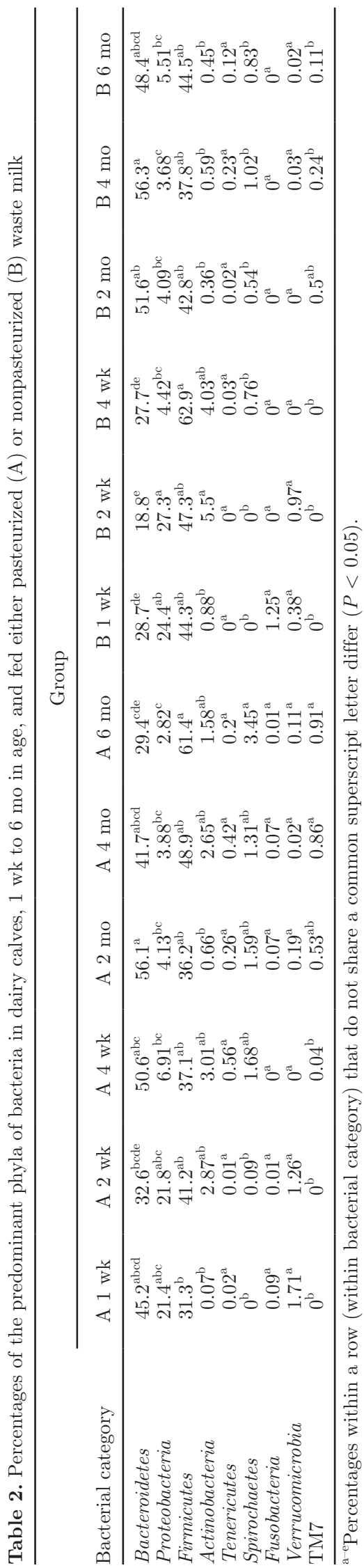

ent, whereas by wk 4 , the animals fed a pasteurized-milk diet had diverged further from the starting enterotype. By 6 mo, animals fed pasteurized milk had resolved into a tight cluster (more similar populations), whereas the nonpasteurized milk-fed group at 6 mo had more differences in their microbiome among the individual animals.

Salmonella was detected consistently in 1-wk-old animals fed nonpasteurized milk. Nine of the 14-wkold calves (64\%) had detectable levels of Salmonella. None of the animals fed pasteurized milk were positive for Salmonella. Among the rest of the samples, only 4 were positive for Salmonella, 2 from the pasteurized milk-fed older animals (2-6 mo of age) and 2 from the nonpasteurized milk-fed groups.

\section{DISCUSSION}

The goal of a successful calf-rearing program is to minimize the stress associated with calf removal from the dam and subsequent exposure to potentially infectious agents by providing a clean, dry housing environment and a nutritious and palatable diet. Newborn calves during the first few weeks of age are particularly susceptible to a variety of intestinal pathogens including Salmonella, Escherichia coli, rotavirus, and Cryptosporidium. Previously, we reported that young dairy calves and cows in the sick/fresh pen were the most likely classes of dairy animals to be shedding Salmonella (Edrington et al., 2008). Salmonella, although routinely cultured from healthy mature animals (Edrington et al., 2004, 2008; Fitzgerald et al., 2003), appears to be opportunistic in exploiting immature (calves) or impaired (sick and fresh cows) gut microbiota.

Nonsaleable waste milk is often fed to dairy calves in either a pasteurized or nonpasteurized form. Significant reductions in total bacterial counts (Ruzante et al., 2008) as well as specific pathogens such as Salmonella, Mycoplasma, and M. paratuberculosis (Stabel et al., 2004) have been reported following pasteurization. Coupled with the reported prevalence of Salmonella in raw milk and colostrum (Sweeney et al., 1992; Butler et al., 2000; Houser et al., 2008), pasteurization of waste milk would seem to be a logical procedure to ensure calf health and reduce pathogen exposure. However, not all farms pasteurize their waste milk before feeding. Additionally, although significant reductions (90-95\% in some cases) in bacterial counts have been reported immediately following pasteurization, recolonization or recontamination of the milk occurs rapidly (Ruzante et al., 2008). Therefore, although pathogenic bacteria in the waste milk can be eliminated, without proper and consistent postpasteurization milk-handling procedures, the total bacterial load reaching the calves 


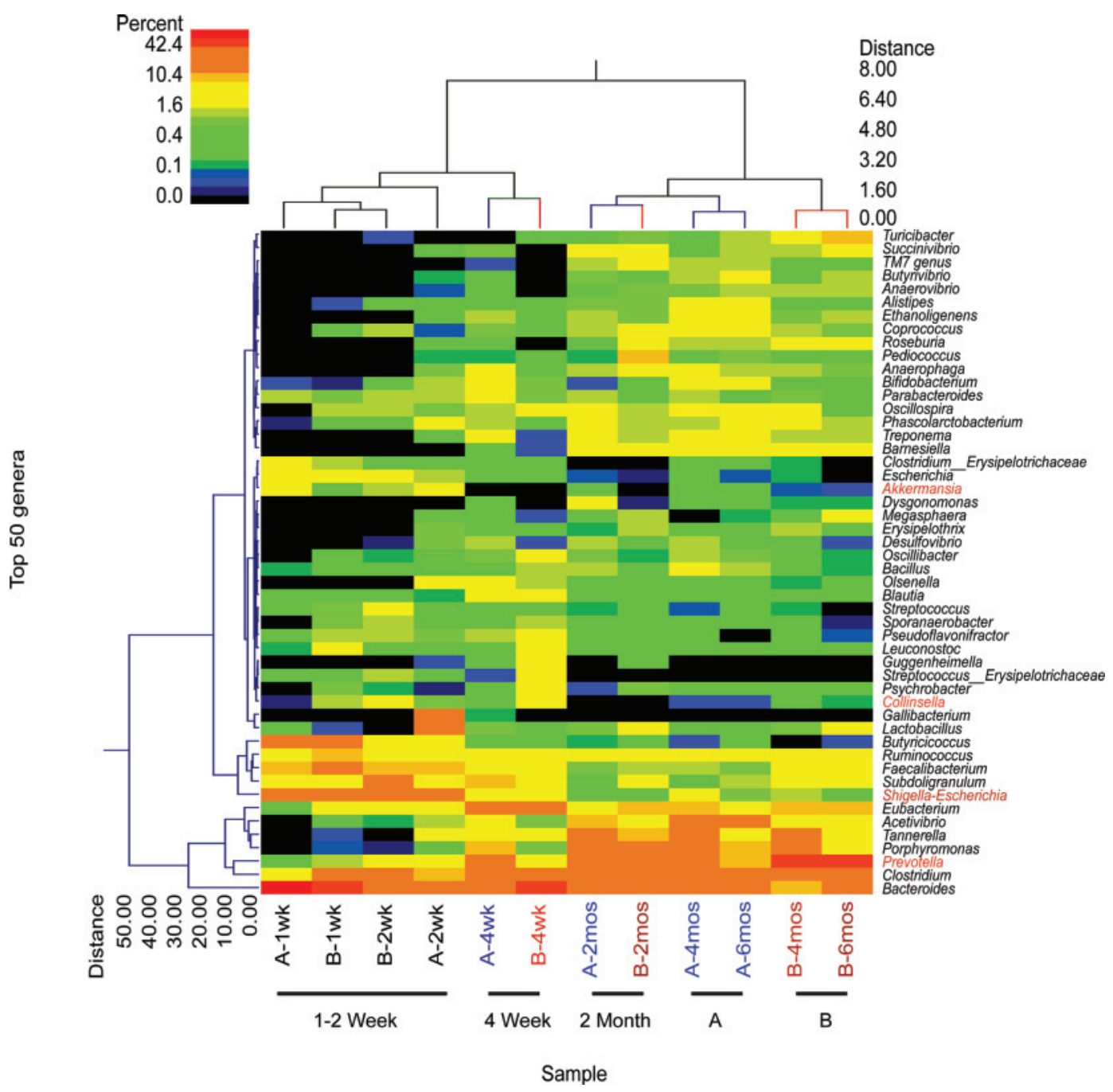

Figure 2. Dendrogram presenting the level of relatedness among fecal samples based upon the 50 most prevalent genera. The percentage of each of the most abundant genera is based upon relative percentage with each group from 2 farms feeding either pasteurized (farm A) or nonpasteurized (farm B) waste milk to calves age 1 wk to 6 mo.

may not be different when comparing pasteurized to nonpasteurized waste milk.

Therefore, the objective of the current research was to determine if pasteurizing waste milk before feeding to dairy calves affected bacterial diversity of the intestinal flora as a measure of gut development. We used a novel tag bacterial diversity amplification method (bTEFAP) that uses massively parallel pyrosequencing techniques and fecal samples from calves (ages 1 wk to $6 \mathrm{mo}$ ) fed either pasteurized or nonpasteurized waste milk. Although biases exist with these molecular approaches compared with traditional culture methods, the data generated by bTEFAP is extensive and provides a very detailed examination of the bacterial microbiome. However, due to the massive amount of data this technique generates, determining the best method of analysis and presentation is not as straightforward as it might seem.
As the animals studied were young calves with developing gut microflora, we chose to look at the overall picture of bacterial diversity as well as a few individual bacterial species of importance in dairy calves.

Overall, the total number of different characterized and noncharacterized bacterial species was greater for calves fed pasteurized waste milk. This was somewhat surprising, as we expected the pasteurization process to provide a cleaner product in terms of bacteria present and, therefore, less diversity. This was the case in calves 1 wk old but not in the older calves. We suspect that the increased diversity in the older calves is likely a result of recolonization of the waste milk following pasteurization. As mentioned above, this occurs rapidly following pasteurization (Ruzante et al., 2008) and may account for the differences in the 2 sets of calves. Unfortunately, we did not sample or culture any of the 


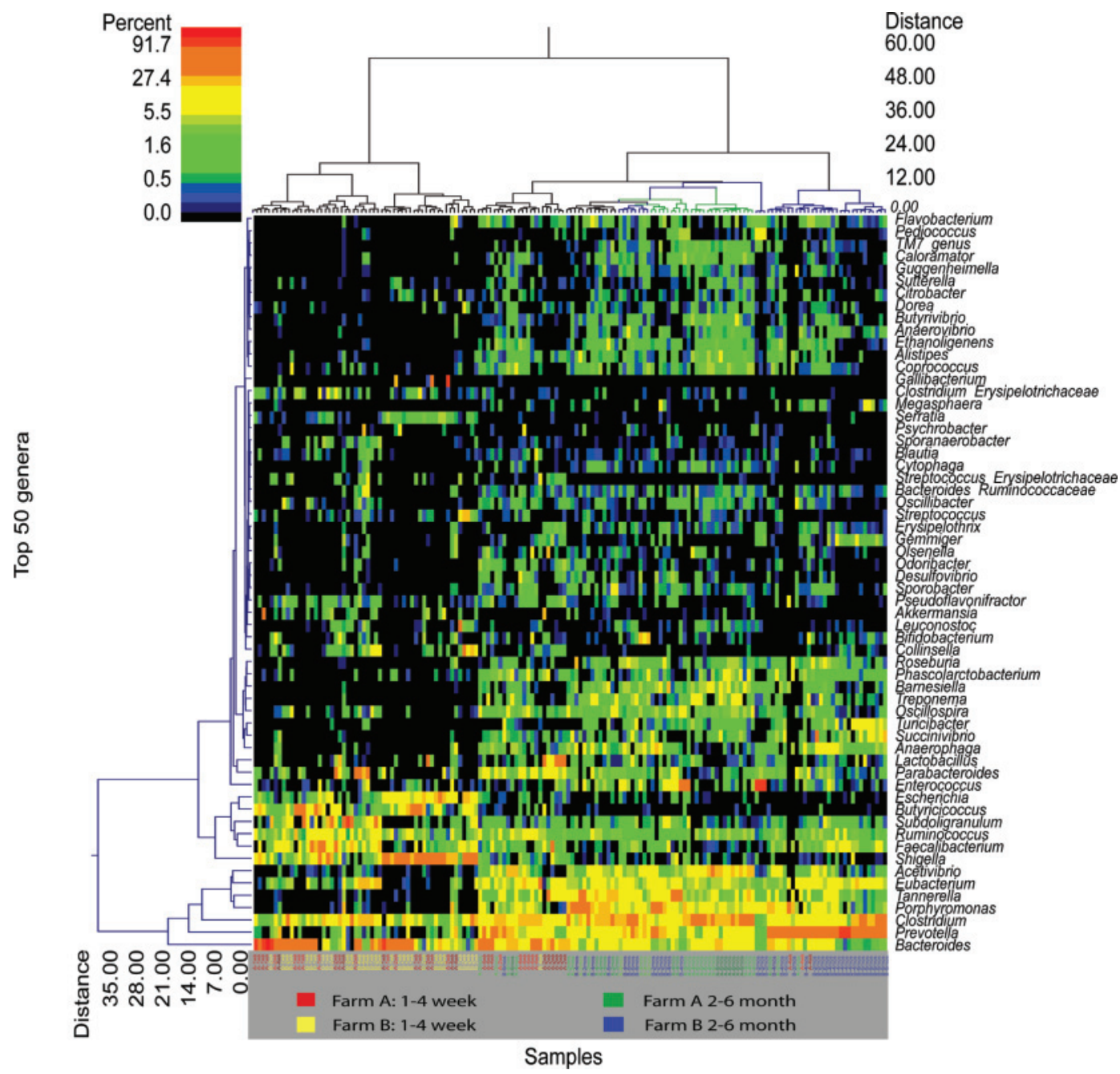

Figure 3. Dendrogram presenting the level of relatedness among fecal samples based upon the 50 most prevalent genera. The percentage of each of the most abundant genera is based upon relative percentage within calves grouped by age (1 to 4 wk of age and 2 to 6 mo of age) from 2 farms feeding either pasteurized (farm A) or nonpasteurized (farm B) waste milk to calves.

waste milk during the study so we can only speculate, but this would seem a logical explanation.

As might be expected, when a phylum was higher in one group, another of the major phyla was typically lower. For example, Bacteroidetes was higher in calves fed pasteurized waste milk at the younger ages, whereas Firmicutes was lower, suggesting the filling of a bacterial void by other phyla. Populations of the lower intestinal bacteria of cattle are dominated by strict anaerobes such as Bacteroides and Clostridium spp. (Drasar and Barrow, 1985), so these results are not surprising. Both groups contain species that can have beneficial and negative effects on the host animal. Analysis of the data via the dendrogram to examine relatedness among bacteria indicated only slight differences due to calf age or pasteurization of the waste milk.
The detection of Salmonella, an important bacterial pathogen in dairy calves, in only the 1-wk-old animals fed nonpasteurized milk was surprising. These data support what has been expressed by dairy producers in numerous personal communications: that Salmonella is a primary concern only in the very young dairy calf. After 1 wk of age, it appears that Salmonella are largely excluded from the intestinal microbiota, or at least their populations are reduced dramatically. We do not know if the young calves with the detectable populations of Salmonella also suffered from clinical manifestations of salmonellosis. Somewhat surprising are the differences in the percentage of Salmonella-positive fecal samples among calves fed the 2 types of waste milk. We hypothesized that feeding pasteurized waste milk would reduce the incidence of Salmonella in calves compared with those receiving nonpasteurized milk. Or, the dif- 
ferences in Salmonella prevalence could be differences among farms as we have reported previously (Edrington et al., 2004). Ideally, we would have preferred to use calves on a single farm and feed them the 2 types of waste milk. However, the reader is reminded that this research was conducted on a commercial dairy farm and in the farm's interest of limiting worker interruptions and maintaining profitability, we were required to make concessions with the experimental design and use 2 different farms. Based on the similarities in Salmonella (expressed as a percentage of the total bacterial population) among milk treatments, it is reasonable to assume that the differences in fecal Salmonella prevalence (\% of Salmonella-positive samples) could also be a factor of farm and not milk source.

Overall, the results of the current research are somewhat surprising in that there were not more consistent differences among calves fed pasteurized or nonpasteurized waste milk. We speculated that nonpasteurized milk would provide a more substantial source of bacteria for gut colonization and subsequently be reflected by a greater bacterial diversity. However, just the opposite was observed and indicates that the recontamination of milk following pasteurization may provide a better source of bacteria for gut establishment. Salmonella populations were similar among treatments, indicating that bacteria provided in both milk sources were effective in reducing Salmonella in the digestive tract of calves. It would also appear from this data that milk-borne Salmonella is not an important vector for transmission to young dairy calves, at least not on these farms. Although the bacterial diversity (as measured by number of different species) data indicate a benefit of pasteurization, the Salmonella data does not show any clear benefit. However, the potential benefits of pasteurization in disease prevention clearly outweigh the potential risks of feeding a nonpasteurized product and should be incorporated into any calf-rearing program using nonsaleable waste milk for feeding young dairy calves.

\section{REFERENCES}

Blosser, T. H. 1979. Economic losses from and the national research program on mastitis in the United States. J. Dairy Sci. 62:119127.

Butler, J. A., S. A. Sickles, C. P. Johanns, and R. F. Rosenbusch. 2000. Pasteurization of discard Mycoplasma milk used to feed calves: Thermal effects on various Mycoplasma. J. Dairy Sci. 83:22852288.

Chardavoyne, J. R., J. A. Ibeawuchi, E. M. Kesler, and K. M. Borland. 1979. Waste milk from antibiotic treated cows as feed for young calves. J. Dairy Sci. 62:1285-1289.

Dowd, S. E., Y. Sun, P. R. Secor, D. D. Rhoads, B. M. Wolcott, G. A. James, and R. D. Wolcott. 2008. Survey of bacterial diversity in chronic wounds using pyrosequencing, DGGE, and full ribosome shotgun sequencing. BMC Microbiol. 8:43-58.
Drasar, B. S., and P. A. Barrow. 1985. The bacterial flora of the normal intestine. Pages 19-40 in Intestinal Microbiology. B. S. Drasar and P. A. Barrow, ed. Am. Soc. Microbiol. Press, Washington, DC.

Edrington, T. S., T. R. Callaway, R. C. Anderson, and D. J. Nisbet. 2008. Prevalence of multidrug-resistant Salmonella on commercial dairies utilizing a single heifer raising facility. J. Food Prot. $71: 27-34$.

Edrington, T. S., M. E. Hume, M. L. Looper, C. L. Schultz, A. C. Fitzgerald, T. R. Callaway, K. J. Genovese, K. M. Bischoff, J. L. McReynolds, R. C. Anderson, and D. J. Nisbet. 2004. Variation in the faecal shedding of Salmonella and E. coli O157:H7 in lactating dairy cattle and examination of Salmonella genotypes using pulsedfield gel electrophoresis. Lett. Appl. Microbiol. 38:366-372.

Fitzgerald, A. C., T. S. Edrington, M. L. Looper, T. R. Callaway, K. J. Genovese, K. M. Bischoff, J. L. McReynolds, J. D. Thomas, R. C. Anderson, and D. J. Nisbet. 2003. Antimicrobial susceptibility and factors affecting the shedding of E. coli O157:H7 and Salmonella in dairy cattle. Lett. Appl. Microbiol. 37:392-398.

Houser, B. A., S. C. Donaldson, S. I. Kehoe, A. J. Heinrichs, and B. M. Jayarao. 2008. A survey of bacteriological quality and the occurrence of Salmonella in raw bovine colostrums. Foodborne Pathog. Dis. 5:853-858.

Ishak, H. D., R. Plowes, R. Sen, K. Kellner, E. Meyer, D. A. Estrada, S. E. Dowd, and U. G. Mueller. 2011. Bacterial diversity in Solenopsis invicta and Solenopsis geminata ant colonies characterized by $16 \mathrm{~S}$ amplicon 454 pyrosequencing. Microb. Ecol. 61:821-831.

Jamaluddin, A. A., T. E. Carpenter, D. W. Hird, and M. C. Thurmond. 1996. Economics of feeding pasteurized colostrums and pasteurized waste milk to dairy calves. J. Am. Vet. Med. Assoc. 209:751-756.

Kesler, E. M. 1981. Feeding mastitic milk to calves: A review. J. Dairy Sci. 64:719-723.

Keys, J. E., R. E. Pearson, and B. T. Weinland. 1980. Performance of calves fed fermented mastitic milk, colostrums, and fresh whole milk. J. Dairy Sci. 63:1123-1127.

Lozupone, C., M. E. Lladser, D. Knights, J. Stombaugh, and R. Knight. 2011. UniFrac: An effective distance metric for microbial community comparison. ISME J. 5:169-172.

Pitta, D. W., W. E. Pinchak, S. E. Dowd, J. Osterstock, V. Gontcharova, E. Youn, K. Dorton, I. Yoon, B. R. Min, J. D. Fulford, T. A. Wickersham, and D. P. Malinowski. 2010. Rumen bacterial diversity dynamics associated with changing from bermudagrass hay to grazed winter wheat diets. Microb. Ecol. 59:511-522.

Ruzante, J. M., I. A. Gardner, J. S. Cullor, W. L. Smith, J. H. Kirk, and J. M. Adaska. 2008. Isolation of Mycobacterium avium subsp. paratuberculosis from waste milk delivered to California calf ranches. Foodborne Pathog. Dis. 5:681-686.

Schloss, P. D., S. L. Westcott, T. Ryabin, J. R. Hall, M. Hartmann, E. B. Hollister, R. A. Lesniewski, B. B. Oakley, D. H. Parks, C. J. Robinson, J. W. Sahl, B. Stres, G. G. Thallinger, D. J. Van Horn, and C. F. Weber. 2009. Introducing mothur: Open-source, platform-independent, community-supported software for describing and comparing microbial communities. Appl. Environ. Microbiol. 75:7537-7541.

Smith, D. M., D. E. Snow, E. Rees, A. M. Zischkau, J. D. Hanson, R. D. Wolcott, Y. Sun, J. White, S. Kumar, and S. E. Dowd. 2010. Evaluation of the bacterial diversity of pressure ulcers using bTEFAP pyrosequencing. BMC Med. Genomics 3:41.

Stabel, J. R., S. Hurd, L. Calvente, and R. F. Rosenbusch. 2004. Destruction of Mycobacterium paratuberculosis, Salmonella spp., and Mycoplasma spp. in raw milk by a commercial on-farm hightemperature, short-time pasteurizer. J. Dairy Sci. 87:2177-2183.

Sweeney, R. W., R. H. Whitlock, and A. E. Rosenberger. 1992. Mycobacterium paratuberculosis cultured from milk and supramammary lymph nodes of infected asymptomatic cows. J. Clin. Microbiol. 30:166-171.

USDA-NASS (US Department of Agriculture-National Agricultural Statistics Service). 2002. Milk cows and production. Accessed Dec. 2002. http://usda01.library.cornell.edu/usda/nass/SB1002/ sb1021.pdf. 